\title{
Paradigmen der vergleichend-historischen Methodologien: Durkheimsche vs. Weberianische Ansätze und ihre Folgen ${ }^{1}$
}

Harold Kerbo

1 Einleitung: Reflexive und vergleichende Soziologie

Soziologen, die sich schon seit den 60er und 70er Jahren mit dem Thema beschäftigen, sahen mit Belustigung $\mathrm{zu}$, wie ihre Kollegen in den Bereichen der freien Künste und der Geisteswissenschaften während der 90er Jahre die „Postmoderne" und den „Dekonstruktivismus" entdeckten. Die Soziologie ist schon seit langem „selbst-reflexiv"; eine "Soziologie der Soziologie“ und eine „reflexive Soziologie" waren schon in den späten 60er und 70er Jahren in aller Munde. Soziologen wie Strasser (1976), Gouldner (1970, 1973) und Friedrichs (1970) halfen uns zu verstehen, wie unerkannte Vorannahmen vielerlei Art unser Denken im Prozess der Forschung und der Theoriebildung umnebeln können. Damit warnten sie uns vor exakt denselben kulturellen und politischen Vorurteilen, von denen die heutigen „Post-Modernisten“ uns erzählen, dass wir sie „dekonstruieren“ müssten.

Ebenfalls während der 60er und 70er Jahre halfen uns Wissenschaftshistoriker wie Kuhn (1962) zu verstehen, welchen Einfluss ,paradigmatische Vorannahmen", die nicht benannt und oft nicht erkannt werden, selbst in den harten Naturwissenschaften, etwa in der Physik, haben. Im Gegensatz zu der idealisierten Auffassung von wissenschaftlicher Methode und Theorie sind die Ansicht eines Wissenschaftlers von dem Untersuchungsgegenstand und die Theoriebildung nicht einzig und allein auf eine kühle Auswertung der verfügbaren empirischen Daten gegründet. Vielmehr müssen Wissenschaftler in ihrer Arbeit bis zu einem gewissen Grad von einem Set von vorwissenschaftlichen und ungeprüften Vorannahmen über die zu erforschenden Phänomene ausgehen. Albert Einstein formulierte das so: „Die bloße Ansammlung erfasster Phänomene reicht niemals aus, um eine Theorie aufzustellen; es muss immer noch eine freie Erfindung des menschlichen Geistes hinzukommen, die zum Kern der Sache vorstößt" (zitiert nach Dukas/Hoffman 1979: 24). Gelegentlich ging Einstein noch darüber hinaus, indem er die Idee zurückwies, dass "Tatsachen aus sich heraus, ohne die

' Übersetzung: Stefanie Osthof 
freie Begriffskonstruktion, wissenschaftliches Wissen hervorbringen können und sollten" (zitiert nach Clark 1971: 63).

In der zweiten Auflage seines Bahn brechenden Werkes nahm Kuhn (1970) auch die Frage nach den Paradigmen in den Sozialwissenschaften auf. Seine nunmehr akzeptierte Ansicht war, dass viel mehr paradigmatische Vorannahmen die Arbeit der Soziologen beeinflussen als in den Naturwissenschaften. Eine größere Zahl der Forschungsgegenstände von soziologischem Interesse ist beladen mit kulturellen, politischen, Klassen- und religiösen Vorurteilen, die sich in unseren Köpfen während des Sozialisierungsprozesses angehäuft haben. Wenige Nicht-Wissenschaftler haben vorgefasste Meinungen darüber, wie sich Quarks und andere subatomare Partikel verhalten; fast jeder wächst hingegen in einer Kultur auf, die ihm „sagt“, warum Menschen Verbrechen begehen, arm oder Terroristen sind.

Die früheren Arbeiten von Strasser $(1976)$, Gouldner $(1970,1973)$ und anderen über soziologische Paradigmen beschäftigten sich überwiegend mit Wertungen, politischen Vorannahmen oder einfach mit allgemeinen Vorstellungen von Gesellschaften; z. B. die Vorannahmen des funktionalen vs. des Konfliktparadigmas. Aber sie waren sich alle der vielen anderen Typen von paradigmatischen Vorannahmen wohl bewusst, die unsere Forschung und Theoriebildung beeinflussen können.

Im vorliegenden Beitrag geht es vor allem um die konkurrierenden vergleichend-historischen methodologischen Ansätze in der Geschichte der Soziologie. Nach einer Untersuchung dieser zwei konkurrierenden methodologischen Ansätze werde ich mich damit beschäftigen, wie die dominantere Durkheimsche vergleichende Methode für Soziologen, Ökonomen und andere Sozialwissenschaftler zu Problemen bei dem Versuch geführt hat, heute den Prozess der ökonomischen Entwicklung und der Verringerung der Armut in den weniger entwickelten Ländern zu verstehen. Außerdem beschäftige ich mich damit, wie diese Methode es erschwert, ein richtiges Verständnis von der genauen Art der sozialen Schichtung in Japan im Vergleich mit den anderen post-industriellen Gesellschaften zu entwickeln. Abschließend werde ich begründen, warum wir einige grundlegende Lektionen der Forschungsmethodenlehre beherzigen müssen, in denen wir lernten, quantitative und qualitative Methoden soweit wie möglich zu kombinieren; Lektionen, die anscheinend in der vergleichendhistorischen Forschung weitgehend vergessen worden sind.

\section{Konkurrierende vergleichend-historische Methodologien in der Geschichte der Soziologie}

Von Beginn des 20. Jahrhunderts an entwickelte sich die amerikanische Soziologie zu einer sich selbst beäugenden, von den anderen Wissenschaften abge- 
schnittenen und ziemlich a-historischen Wissenschaft. Vergleichend-historische Methodologien wurden, um es vorsichtig auszudrücken, selten angewendet. Erst langsam drang die globale Welt nach dem Zweiten Weltkrieg weiter ins Bewusstsein der amerikanischen Soziologie, und bedeutende Theorien wie der Funktionalismus begannen, eine stärker vergleichend-historische Perspektive einzunehmen. Das Streben der amerikanischen Soziologen nach wissenschaftlichem Status bedeutete jedoch, dass sie "sich wissenschaftlicher darstellen“" wollten, und das bedeutete: immer mehr statistische Analyse. Bis in die jüngste Vergangenheit hinein bedeutete diese statistische Analyse für gewöhnlich die Verteilung von hunderttausenden Fragebögen oder die Gewinnung von Individualdaten durch Erhebungen der Regierung. Bei Ausdehnung der zu analysierenden Einheit auf ein nationales Niveau wurden die „Ns" zu klein für die damaligen statistischen Methoden. Selbst unter Einbeziehung einiger Zeitdimensionen (oder „Ts") für jedes Land in die Analyse blieben die „Ns" $\mathrm{zu}$ klein für die anerkannten Methodologien. Um „wissenschaftlich" zu bleiben, hielten sich die amerikanischen Soziologen deshalb an den Einzelnen als zu analysierende Einheit, wenngleich der Wirkungsbereich der Soziologie ein weiteres Feld der Analyse hätte umfassen sollen.

Von den 70er Jahren an entwickelte oder erkannte die Soziologie jedoch neue statistische Methoden, zuerst in den Vereinigten Staaten, dann auch in Europa, welche weniger Fälle (oder "Ns") erforderten und Autokorrelation von graduellen Veränderungen über historische Zeitabschnitte hinweg kontrollieren konnten. Mit dem Vordringen der Zeitreihenanalyse waren einige der Probleme überwunden worden, die die statistische Analyse in der Soziologie auf den Einzelnen als Analyseeinheit beschränkten. Die typische vergleichend-historische Methodologie umfasst heute ein Sample von 25 bis 50 oder sogar noch mehr Nationen oder Untereinheiten quer durch alle Nationen. Es werden Daten zu einer Reihe von unabhängigen Variablen gesammelt, wie z. B. das Ausmaß von ausländischen multinationalen Wirtschaftsinvestitionen in jedem Land, die Höhe ausländischer finanzieller Hilfe, die jedem Land zufließt, ausstehende Schulden, die an reichere Nationen und internationale Agenturen gezahlt werden müssen, Handelsströme usw. Dann werden Daten zu abhängigen Variablen gesammelt, indem so etwas gemessen wird wie das Wachstum, ausgedrückt durch das Bruttosozialprodukt, Einkommensungleichheit und verschiedene Indikatoren für den Lebensstandard wie Armutsniveaus, Lebenserwartung und Kindersterblichkeit. Verzögerte Zeitintervalle von 5 oder 10 Jahren werden verwendet, in denen den unabhängigen Variablen (etwa den multinationalen Wirtschaftsinvestitionen) Zeit gelassen wird, ihre theoretisch erwarteten Effekte zu bewirken; dann werden statistische Korrelationen gebildet mit dem Ziel herauszufinden, ob zwischen diesen Variablen Zusammenhänge gefunden werden können (wie 
z. B. der Zusammenhang von internationalen Wirtschaftsinvestitionen und höheren Niveaus von Einkommensungleichheit nach 10 Jahren). Die neue vergleichend-historische Methode hat z. B. Soziologen, die sich für soziale Ungleichheit interessieren, geholfen, unterschiedliche oder gleiche politische und soziale Kräfte zu verstehen, die Nationen übergreifend auf die Raten von sozialer Mobilität oder Einkommensungleichheit einwirken (Wright 1997; Erikson/Goldthorpe 1992; Alderson/Nielsen 2002). Weltsystemtheoretiker haben diese neue vergleichend-historische Methodologie genutzt, um die negativen Effekte der wirtschaftlichen Abhängigkeit für weniger entwickelte Nationen in der Weltwirtschaft zu verstehen (Bornschier/Chase-Dunn 1985; Firebaugh 1996, 1992).

Diese Art von Forschung war schon immer komplex, weil es zu Kontroversen um die korrekten Maße der Variablen kommen kann. Trotz dieser Schwierigkeiten haben 30 Jahre Forschung mit den neuen vergleichend-historischen Methodologien in Bereichen wie soziale Ungleichheit und das moderne Weltsystem beeindruckende Ergebnisse hervorgebracht. Wir sind heute beispielsweise ziemlich sicher, dass unterschiedliche Grade von gewerkschaftlicher Organisation und der Nähe von Regierungen zur kapitalistischen Klasse quer durch alle Industrienationen hinweg signifikante Effekte auf die Grade von Einkommensungleichheit und Armut haben (Moller et al. 2003). Wir wissen, dass ein hohes Niveau von Auslandsinvestitionen von einem einzelnen reichen Land die Ursache für weniger Wirtschaftswachstum in ärmeren Ländern sein kann (Kentor/Boswell 2003).

Aber uns muss klar sein, was diese Forschung gezeigt hat und was sie nicht gezeigt hat, und wir müssen klar die inhärenten Beschränkungen der aktuellen vergleichend-historischen Methodologien benennen. Häufig wird übersehen, dass ein statistisch signifikanter Zusammenhang zwischen etwa multinationalen Investitionen zu einem Zeitpunkt und langsamerem Wirtschaftswachstum zu einem späteren Zeitpunkt (10 Jahre später) einfach nur bedeutet, dass in den meisten Nationen mit großen Beträgen von multinationalen Wirtschaftsinvestitionen die ökonomischen Wachstumsraten später abgesunken sind. Es bedeutet nicht, dass dies in allen Nationen geschieht, vielmehr lediglich, dass so etwas tendenziell bei der Durchsicht des Samples von 50, 75 oder 100 Nationen auftritt. Das bedeutet natürlich, dass einige Nationen in dem Sample ein hohes Niveau an ausländischen multinationalen Wirtschaftsinvestitionen und ein starkes Wirtschaftswachstum in späteren Jahren haben. Recht häufig werden die Autoren dieser Forschungsrichtung anmerken, dass einige (manchmal nicht einmal genau benannte) Länder „Ausreißer“ seien, was bedeutet, dass sie außerhalb der Bereiche liegen, in denen sich die anderen Länder gemäß den Variablen zu einer Linie versammeln. Aber damit ist dann das Thema erledigt. Warum sind diese Länder „Ausreißer"? Was ist anders an ihnen, dass sie sich nicht in den sta- 
tistisch signifikanten Zusammenhang zwischen den wichtigen untersuchten Variablen einfügen?

Zum Zweiten müssen wir erkennen, dass die Methodologien, die überwiegend in der Weltsystemforschung angewendet werden, einer Durkheimschen Tradition folgen (Ragin/Zaret 1983; Smelser 1976). Es war natürlich Durkheim, der als Erster annahm, dass es alle Gesellschaften überspannende „natürliche Gesetze" des menschlichen Verhaltens und der sozialen Organisation gebe, und der dann in seinem meisterhaften Werk Der Selbstmord (Suicide, 1857 [1951]) zeigte, wie diese „Gesetze" aufgedeckt werden können. Anhand dieser Durkheimschen Methodologie kann die Forschung beispielsweise wichtige Tendenzen über Nationen hinweg aufdecken, aber gleichzeitig bleiben viele Details darüber, was innerhalb der einzelnen Nationen passiert, im Dunkeln. Gegen diese Methodologie wurden neuerdings Vorwürfe laut; die Weltsystemforschung übersehe in Nationen ,interne Prozesse“, die dafür mitverantwortlich seien, dass bei diesen Nationen trotz des gleichen Niveaus an ausländischen multinationalen Investitionen verschiedene Ergebnisse erzielt würden (Alderson/Nielsen 1999).

Mit dieser „Durkheimschen“ vergleichenden Methodologie können Soziologen immer mehr Variablen mit aufnehmen, Maße verbessern und ,robustere“ Modelle bauen, um den Betrag der erklärten Varianz zu erhöhen. Das Bestreben ist, in anderen Worten, bessere Modelle zu bauen, die Nationen übergreifend zu dem Datensatz passen. Solch ein Bestreben ist ohne Zweifel wichtig dafür, unser Verständnis der menschlichen Gesellschaften zu verbessern. Aber es besteht die Gefahr, dass Kombinationen historischer Kräfte oder Kombinationen von Variablen, die möglicherweise für bestimmte Nationen einzigartig sind, oft unberücksichtigt bleiben.

Im Gegensatz dazu wies die vergleichend-historische Forschung von Max Weber solche eindimensionalen Gesetze der Durkheimschen Perspektive zurück und verlangte stattdessen die Anerkennung komplexer und ziemlich einzigartiger Kombinationen historischer Kräfte, die durch ihr Zusammenwirken wichtige Ergebnisse für jedes Land produzieren. Noch weitergehend erkannte Weber an, dass bestimmte Ergebnisse sogar in unterschiedlichen Ländern verschiedene Gründe haben könnten (Smelser 1976: 142). Es liegt auf der Hand, dass bis zu dem Grad, zu dem das zutriff, eine qualitative vergleichende und historische Analyse von bestimmten Nationen oder von Gruppen relativ ähnlicher Nationen sich am besten dazu eignet, wichtige kausale Faktoren zu entdecken, die nicht in Nationen überall auf der Welt zu finden sind (Ragin 2000). Mit „qualitativer“ Forschung wird eine Forschung bezeichnet, die sich nicht vorrangig auf Zahlen oder solche Dinge stützt, die leicht über viele Nationen hinweg gemessen und verglichen werden können. Der Forscher muss sich bemühen, spezifischere De- 
tails über jeden einzelnen untersuchten Fall herauszufinden (wie z. B. den wirtschaftlichen Entwicklungsstand einer Nation), bevor er versucht, Vergleiche mit anderen Nationen anzustellen, die ebenfalls mit qualitativen Forschungsmethoden untersucht wurden.

Dies soll nicht bedeuten, dass vergleichend-historische Forschung, die aus einer Durkheimschen Perspektive über Nationen hinweg mit Zeitreihenanalysen arbeitet, aufgegeben und frühere Ergebnisse verworfen werden sollten. Aus den letzten 30 Jahren existieren z. B. beeindruckende Aufzeichnungen empirischer Forschung über Themen im Zusammenhang mit der modernen Weltsystemtheorie und der ökonomischen Entwicklung.

Vieles konnte über die Auswirkungen in Erfahrung gebracht werden, die ein eingenommener Rang in diesem Weltsystem für bestimmte Länder und die Menschen in diesen Ländern hat. In der zweiten Welle der Forschung zum Weltsystem in den späten $80 \mathrm{er}$ und frühen 90er Jahren wurde auch vieles über die internen Prozesse in Erfahrung gebracht (wie z. B. die Staatsform, der Handelstypus, das Niveau des Humankapitals). Diese Variablen stehen etwa mit dem Rang im Weltsystem und dem Niveau der ausländischen multinationalen Wirtschaftsinvestitionen in Wechselwirkungen und produzieren so Ergebnisse wie mehr oder weniger ökonomische Entwicklung oder mehr oder weniger Einkommensungleichheit. Um jedoch ein besseres Verständnis davon zu bekommen, wie das moderne Weltsystem die Aussichten auf Verringerung der Armut oder auf ökonomische Entwicklung für die Massen der Welt beeinflusst, scheint es so, als ob eine dritte Forschungswelle eine viel größere Anzahl von Fallstudien einschließen sollte, die mit einer mehr an Weber orientierten qualitativen vergleichenden und historischen Analyse arbeiten.

\section{Westliche Voreingenommenheit in der soziologischen Theorie}

Während die zurzeit vorherrschende vergleichende Methodologie im Sinne des Durkheimschen Paradigmas zwar keine westliche Voreingenommenheit in der soziologischen Theorie hervorbrachte, trägt sie gleichwohl zu dieser Voreingenommenheit bei. Viele, wenn nicht sogar die meisten der "Ausreißer" in den internationalen Datensets sind asiatische Nationen. Diese Art von Methodologie ist auf die Überprüfung von Modellen und Theorien ausgerichtet und nicht gut dazu geeignet, einzigartige Charakteristiken von Nationen oder Nationengruppen zu erkennen. Zum Beispiel deuten Daten der Weltbank und anderer internationaler Agenturen darauf hin, dass trotz (oder, wie die Weltbank behauptet, wegen) extensiver Auslandsinvestitionen einiger Kern-Nationen die ökonomische Entwicklung und die Verringerung der Armut in Ost- und Südostasien im Vergleich mit Afrika oder Lateinamerika viel größer sind (Weltbank 2000: Kapitel 3, S. 45). Aber obwohl solche Informationen seit Jahren vorliegen, wird 
das fast nie in der modernen Weltsystemtheorie und der damit einhergehenden Forschung erwähnt.

Man gewinnt oftmals den Eindruck, dass es westliche Sozialwissenschaftler vollkommen verblüfft, wenn sie mit asiatischen Nationen konfrontiert sind, die scheinbar nicht „passen“. Die asiatischen „Ausreißer" stechen manchmal stark hervor. Ahnungslos hinsichtlich der internen Dynamik asiatischer Nationen merken diese Sozialwissenschaftler für gewöhnlich lediglich an, dass diese anders seien, und gehen dann zu anderen Themen über.

In seinem neuen Buch ReOrient: Global Economy in the Asian Age zitiert Frank (1998: 7-9, 323) Marx, Weber, Simmel, Durkheim und Sombart als zentral für die Entwicklung eurozentristischer soziologischer Theorie. Marx wird beispielsweise kritisiert für sein Konzept des asiatischen Modus, als einer rigiden Form politischer Herrschaft, die nur den Interessen von Eliten diene und sich nicht in die asiatische Tradition einfüge, nach der politische Macht mit der vergleichsweise strengen Beschränkung selbstsüchtiger Ausbeutung von Bürgern durch Eliten einhergeht. Genau wie Weber war auch Marx falsch über die chinesische Geschichte informiert und ließ viele Umbruchs- und Entwicklungszeiten unter den Tisch fallen. Aber in Übereinstimmung mit den Kritikern, die der westlichen Wirtschaftstheorie vorwerfen, bei dem Versuch versagt zu haben, den Aufstieg der japanischen Wirtschaftskraft im 20. Jahrhundert zu verstehen, wendet Frank den Hauptteil seiner Kritik gegen Webers Ansicht, nach der die Entwicklung des Kapitalismus individualistischen Prinzipien folge, die durch die protestantische Ethik ermöglicht würden. Kurz gesagt findet sich scheinbar kein westliches theoretisches oder ideologisches Schema der Dinge, in das die gegenwärtige ostasiatische Erfahrung besonders gut hineinpasste. Im Gegenteil scheint das, was im Moment in Ostasien passiert, alle Arten westlicher Lehren darüber zu verletzen, wie etwas, eigentlich' gemacht werden sollte. Hier wird der Anspruch des Westens deutlich, die, westliche Art' sei zu kopieren (vgl. Frank 1998: 7).

Was die großen Soziologen früherer Zeit angeht, so war ein Großteil ihrer voreingenommenen Sicht auf Asien zurückzuführen auf Unwissenheit und Desinformation; sie lagen einfach falsch in Bezug auf viele grundlegende Tatsachen asiatischer Gesellschaften. Beispielsweise schien keiner von ihnen zu wissen, dass China vor über 500 Jahren technologisch und wirtschaftlich im Vergleich mit Europa viel weiter entwickelt war, oder dass China von Ostafrika über ganz. Ostasien hinweg wirtschaftlich dominant war (Levathes 1994; Frank 1998). Aber gerade diese Unwissenheit trug dazu bei, dass sie ihre Theorien mit westlichen Vorurteilen bildeten, die sie nicht durchschauen konnten.

Max Weber war natürlich einer der wichtigsten frühen Soziologen, die versuchten, ökonomische Entwicklung zu erklären. Nach Weber konnte Japan sich 
nicht aus sich heraus wirtschaftlich entwickeln und tat es auch nicht, sondern die wirtschaftliche Entwicklung musste aus dem Westen importiert werden (Golzio 1985; Yawata 1963). Aufbauend auf seiner These in Die protestantische Ethik und der Geist des Kapitalismus und zudem auf seinen Vorstellungen darüber, warum China keine wirtschaftliche Entwicklung erreichen könne, argumentierte Weber, dass es drei Hauptgründe dafür gebe, dass Japan sich nicht früher habe industrialisieren können:

1) Die japanische Klasse der Kaufleute habe im feudalen Standessystem Japans einen extrem niedrigen Status innegehabt und sei in einem solchen Ausmaß kontrolliert worden, dass sie vom freien Handel und von der Möglichkeit zu prosperieren abgeschnitten gewesen sei.

2) Weber argumentierte auch, dass es in Japan an einem städtischen Umfeld fehle, in dem eine Klasse von Kaufleuten sowie nicht-agrarische ökonomische Aktivität entstehen könnten.

3) Schließlich nahm Weber an, dass die japanischen Hauptreligionen (Buddhismus und Shinto) den Kapitalismus nicht so förderten, wie er es vom Protestantismus in Nord-West-Europa annahm.

Wir wissen heute, dass Weber Unrecht hatte, besonders im Hinblick auf die letzten beiden Punkte, und, darüber hinaus, dass Weber auch Unrecht hatte in Bezug auf den Mangel an ökonomischer Entwicklung im Japan der TokugawaZeit (der Zeitspanne von ungefähr 250 Jahren, in denen Japan gegen andere Nationen relativ abgeschottet war). Die neueste Geschichtsforschung hat Hinweise auf viel mehr ökonomische Entwicklung in Japan gegeben als Webers westlichen Zeitgenossen bekannt war, und deshalb kann nicht behauptet werden, dass Japan sich nur wegen Interventionen von außen hätte entwickeln können (Reischauer/Craig 1978: 94-98; Collins 1997). Tatsächlich begannen einige der großen Handelshäuser, die später zur zaibatsu-Unternehmensgruppe und zu den größten Finanzfirmen werden sollten, wie etwa Mitsui und Nomura, ihre Entwicklung während der Tokugawa-Herrschaft und legten schon die Grundlage für industrielle Expansion. Es kann kaum behauptet werden, dass die Klasse der Kaufleute in Japan unterdrückt und am Wachstum gehindert worden wäre, wenn wir erkennen, dass gegen Ende der Tokugawa-Zeit im Jahre 1868 diese unterdrückte Klasse der Kaufleute über 95 Prozent allen Vermögens in Japan kontrollierte (Halliday 1975: 6; siehe auch Golzio 1985: 93; Alletzhauser 1990).

Was die japanische Religion angeht, machten Weber und seine Quellen die übliche unrichtige Annahme, dass das Meiste der japanischen Kultur von China importiert worden wäre und deshalb der Buddhismus wie auch der Konfuzianismus in Japan Anschauungen enthielten ähnlich den Anschauungen in diesen Religionen in China. Das bedeutete für ihn auch, dass diese Anschauungen die weltliche ökonomische Aktivität hemmen würden. Aber Weber erkannte nicht 
die Verschiedenartigkeit der buddhistischen Sekten, die sich in Japan entwickelten. Einige von ihnen konnten die ökonomische Entwicklung unterstützen und taten das auch (Golzio 1985: 98; Collins 1997). Genauso wenig erkannte Weber, wie Bellah dargelegt hat, dass die verschiedenen japanischen Arten des Buddhismus, des Konfuzianismus und der in Japan einheimischen ShintoReligion sogar so betrachtet werden konnten, dass sie Webers Hauptthese unterstützten, da sie einen Unterbau für die ökonomische Entwicklung im Japan der Tokugawa-Zeit bereit stellten (Bellah 1985).

Bis vor kurzem war die westliche soziologische Theorie keiner dieser kritischen Überprüfungen unterzogen worden. Schließlich nahm Frank (1998) den Vorwurf der westlichen Voreingenommenheit auch in diesem Forschungsbereich auf. Aber Frank zeigte dabei vor allem, wie westliche Soziologen fast vollständig die Tatsache vernachlässigt haben, dass ein mächtiges asiatisches Weltsystem lange vor dem eurozentristischen modernen existierte und das spätere eurozentristische System sogar viele Aspekte des früheren asiatischen kopierte. Im Geiste einer "Soziologie der Soziologie", die lange vor dem heute verbreiteten „postmodernen Dekonstruktivismus" existierte, müssen wir uns der Möglichkeit stärker bewusst werden, dass westliche Annahmen viele unserer spezifischen Theorien über Themen wie die Gründe für Suizid, soziale Mobilität oder Geschlechterverhältnisse, um nur einige zu nennen, einseitig beeinflusst haben.

Greift man einmal den Bereich der ökonomischen Entwicklung heraus, so scheinen hier die folgenden Punkte von größter Bedeutung zu sein (Kerbo 2006a):

1) Die gängigen Theorien haben übersehen, wie asiatische Traditionen der Autorität und der staatlichen Organisation sich zu politischen Strukturen zusammenfügen, die besser geeignet sind, allgemeine nationale Interessen zu schützen, multinationalen Unternehmen die Stirn zu bieten und Eliten im Zaum zu halten, sodass eher die allgemeinen nationalen Interessen gesichert werden als einige auf einheimische Eliten beschränkte Einzelinteressen.

2) Westliche individualistische Vorannahmen bringen Sozialwissenschaftler häufig zu der Schlussfolgerung, dass eine nach westlichen Definitionen weniger demokratische Gesellschaft automatisch zur Ausbeutung durch die Eliten aus deren selbstsüchtigem Interesse heraus führt; diese Vorannahmen bringen uns dazu, andere traditionelle Mechanismen der Beschränkung von Eliten in asiatischen Gesellschaften außer Acht zu lassen.

3) Westliche, besonders angloamerikanische Werte des Individualismus und des Misstrauens gegenüber dem Staat haben westliche Ökonomen und viele Theoretiker des Weltsystems dazu gebracht außer Acht zu lassen, bis zu welchem Grad staatliche Intervention solide ökonomische Entwicklung bewirken kann. 


\section{Soziale Stratifikation in Japan: Ein Beispiel für westliche Voreingenommenheit}

Wie oben erwähnt wurden in der vergleichend-historischen Forschung einige beeindruckende Erfolge erzielt, was die soziale Mobilität quer durch alle modernen Industrienationen angeht (z. B. Grusky/Hauser 1984; Erikson/Goldthorpe 1992). Wir haben viel erfahren über jene Bedingungen, vor allem die Grundzüge der Wirtschaft und die politischen Maßnahmen einer Nation, welche die Mobilitätsraten heben oder senken. Wenn jedoch wichtige Dimensionen sozialer Stratifikation nicht adäquat gemessen werden oder nicht adäquat in jene Modelle eingefügt werden, die Mobilitätsraten voraussagen, sind - und waren die Ergebnisse irreführend.

Als das erste vollkommen industrialisierte Land in Asien ist Japan ein wichtiger Testfall für westliche Vorannahmen darüber, wie die wirtschaftliche Entwicklung ablaufen kann und wie die wichtigsten Institutionen eines Landes aussehen müssen, wenn es den vollen Status einer Industrienation erreicht. Einer der Modernisierungstheoretiker der ersten Stunde, der in den frühen 50er Jahren mit Talcott Parsons gearbeitet hatte, räumte kürzlich ein, dass sie mit vielen Erwägungen falsch gelegen hätten, wenn man bedenke, wie das moderne Japan sich bis zur Gegenwart entwickelt habe (Bellah 1985). Ich werde hier aber nur die soziale Schichtung in Japan als ein Beispiel dafür betrachten, wie unsere vergleichend-historische quantitative Forschung aus einer mehr Durkheimschen Perspektive uns irreführen kann.

In westlichen industriellen und post-industriellen Gesellschaften gehen Theorien sozialer Ungleichheit davon aus, dass von Webers Dimensionen Klasse, Status und Macht die Dimension der Klasse dominant geworden sei. Als Folge davon hat sich der größte Teil der empirischen Ungleichheitsforschung in den modernen industriellen Gesellschaften der sozialen Mobilität zugewandt (für eine Zusammenfassung dieser Forschungstrends siehe Kerbo 1981, 2006b). Und bis vor gar nicht allzu langer Zeit drehten sich alle Maße sozialer Mobilität um Positionen des beruflichen Status. Heute gibt es den neuen Trend, Einkommensmobilität über Generationen hinweg zu messen, statt nur die berufliche Mobilität, aber selbst hier liegt der Schwerpunkt ausschließlich auf der ökonomischen Stellung (Hertz 2004; Kerbo 2006b: 376). Die Frage für die vergleichende Forschung, insbesondere im Hinblick auf asiatische Gesellschaften wie Japan, muss lauten: Sind die Berufs- und Einkommensdimensionen in den modernen Systemen sozialer Ungleichheit gleich wichtig? Wissenschaftler mit umfassenden Erfahrungen in Japan stellen diese Annahme in Frage und argumentieren, dass Webers Statusdimension nach wie vor besteht. Qualitative vergleichende Forschung eignet sich am besten dazu, Fragen zur Anwendbarkeit unserer Konzepte und Maße zu beantworten, und sollte begleitend zur oder sogar vor 
der quantitativen historischen und vergleichenden Forschung betrieben werden. Es folgt ein kurzes Beispiel dazu, was eine qualitative vergleichende Analyse sozialer Ungleichheit berücksichtigen sollte.

\section{Anomalien im japanischen System der sozialen Schichtung}

Belege für eine westliche Voreingenommenheit in der aktuellen soziologischen Forschung zu Japan und zu einigen besonderen Aspekten der sozialen Ungleichheit in Japan (im Vergleich mit westlichen Industrieländern, nicht notwendigerweise im Vergleich mit anderen asiatischen Ländern) drängen sich auf bei der Betrachtung von gewissen - wie ich sie nennen möchte - „Anomalien" im japanischen Schichtungssystem. Einigen dieser Anomalien wende ich mich nun für eine kurze Betrachtung zu.

Bürokratische Autorität und Status. Ein Thema, das die gesamte historische und gegenwärtige wissenschaftliche Untersuchung der japanischen Gesellschaft, Wirtschaft und des japanischen politischen Systems durchzieht, ist der große Respekt und Status, der Amtsträgern der Regierungsbürokratie eingeräumt wird (siehe z. B. Koh 1989; Park 1986; Johnson 1982; Eisenstadt 1996; Jansen $2000)^{2}$. Der Grad der rituellen Ehrerbietung, die diesen Beamten in der sozialen Interaktion entgegengebracht wird, die beliebten Romane, die über „bürokratische Helden" geschrieben werden, Zeitschriften, die sich auf das Leben der obersten Bürokratiebeamten spezialisieren, und der Begriff amakudari (,vom Himmel herabsteigen"), der benutzt wird, um Bürokratiebeamte zu beschreiben, die in die obersten Etagen von Unternehmen ,hinab"-steigen, können als qualitative Belege eines solchen Status angegeben werden (Colignon/Usui 2003). Ein weiterer Hinweis auf den Status der Bürokratiebeamten ist der Gebrauch des Begriffs kanryodo, der mit dem Begriff bushido, „Wesensart des Kriegers“ oder „Wesensart des Samurai“ verglichen werden muss. Das kanji-Schriftzeichen (chinesisches Schriftzeichen), das "do" ausgesprochen wird, bezieht sich auf einen ehrbaren oder geachteten Lebenswandel und eine Lebensphilosophie. Dieser neue Begriff kanryodo wird im modernen Japan also übersetzt mit „die Wesensart des Bürokraten" (Johnson 1982; Koh 1989).

Ein vielleicht noch besserer Indikator für den Status dieser ministerialen Positionen ist die Tatsache, dass sie in der Lage sind, viele, wenn nicht die meisten, der besten Absolventen von den am höchsten geachteten Universitäten Japans zu bekommen, obwohl die Entlohnung, die für die Beschäftigung auf diesen bürokratischen Posten geboten wird, weit unterhalb der Entlohnung liegt,

\footnotetext{
${ }^{2}$ Mit Regierungsbürokraten sind Beamte der Ministerien der Regierung gemeint. Gewählte Politiker im Parlament haben z. B. keinen solchen Status. Und der Status dieser Regierungsbürokraten wird natürlich vom Rang innerhalb des Ministeriums bestimmt, jedoch auch von dem Ministerium, in dem sie beschäftigt sind (Koh 1989).
} 
die im Unternehmenssektor festgestellt werden kann, welcher lebhaft versucht, diese Absolventen anzuwerben (Koh 1989; Colignon/Usui 2003).

Nach allgemeiner Auffassung ist es wahrscheinlich, dass der Status von Regierungsbürokraten in vielen Industriestaaten nicht annähernd so niedrig ist, wie es in den Vereinigten Staaten der Fall ist. Aber selbst im Vergleich mit solchen Ländern wie Frankreich wird der Status von Regierungsbürokraten in Japan als viel höher bezeichnet, trotz der sehr geringen Bezahlung (Koh 1989; Reischauer 1987; Kerbo/McKinstry 1995; Colignon/Usui 2003). Es trifft zu, dass diese japanischen Ministeriumsbürokraten einiges von diesem Status in den jüngsten Bestechungsskandalen eingebüßt haben, die es vorher fast nicht gab. Aber sie ziehen immer noch die besten Absolventen von Japans Eliteuniversitäten an. Und Japan steht nicht allein unter den asiatischen Ländern, die den Regierungsbürokraten außergewöhnlich hohen Status zubilligen (Pye 1985; Wyatt 1984; Girling 1981). In Thailand werden z. B. Regierungsbürokraten als persönliche Stellvertreter des Königs angesehen, und der thailändische Begriff für den bürokratischen Beamten bedeutet eigentlich „königlicher Diener" (Girling 1981: 147; Keyes 1989). Die Auffassung von Bürokratiebeamten als offiziellen Stellvertretern des Herrschers findet sich auch in Japan; sie wurde sogar von der neuen Meiji-Oligarchie kultiviert als Mittel der Absicherung dafür, dass diese Bürokratie mehr Macht inne hätte als das neue Parlament haben würde, als dieses unter der neuen Verfassung von 1889 errichtet wurde (Gluck 1985). Aber genauso bedeutend ist das Argument, dass im Gegensatz zu den wirtschaftlichen Eliten des privaten Sektors die bürokratischen Eliten als Vertreter von Gruppeninteressen (das heißt nationalen Interessen) - vor persönlichen (das heißt selbstsüchtigen) Interessen - angesehen und deshalb höher geachtet werden müssen. Diesem Faden folgend ist der Hinweis von Nutzen, dass der ursprüngliche japanische Begriff für Geschäftsmann oder Händler eine negative Konnotation von Selbstsucht hatte und mit einem niederen Status in Verbindung gebracht wurde. Während der Meiji-Zeit und der ökonomischen Entwicklung wurde dieser Begriff für Händler geändert; damit wollte man versuchen, den Status der Händler zu verbessern.

Burakumin. Eines der interessantesten Merkmale der Hierarchie in Japan ist heutzutage die fortdauernde Existenz und Diskriminierung von burakumin. Wenigstens ein gewisser Grad von Diskriminierung verschiedener rassischer und ethnischer Gruppen kann in allen industriellen Gesellschaften gefunden werden; auch Japan hat sicherlich seinen Anteil an dieser Art von Diskriminierung, z. B. im Verhältnis zu den $A i n u$-Eingeborenen, ethnischen Chinesen und Koreanern. Aber die Situation der burakumin stellt sich ganz anders dar. Burakumin sind rassisch und ethnisch Japaner, die oft anhand ihrer Erscheinung, Sprache, Familiennamen oder Lebensstil nicht als burakumin erkannt werden können. Das 
macht die burakumin zu einer reinen Statusgruppe ohne rassische oder ethnische Verwicklungen, wie sie bei den Koreanern oder Chinesen in Japan, oder den Schwarzen, Hispano- oder asiatischen Amerikanern in den Vereinigten Staaten gefunden werden können.

Die heutigen burakumin sind die Nachfahren jener Gruppe von Ausgestoßenen, die ursprünglich mit eta (,durch und durch verschmutzt") oder hinin (,nicht-menschlich“) bezeichnet wurden. In der Tokugawa-Zeit wurde der Status dieser Menschen sogar noch herabgesetzt und noch vollkommener vererblich gemacht (Neary 1997; Hane 1982: 139-163; DeVos/Wagatsuma 1966). Es wurde von ihnen verlangt, dass sie in abgetrennten Dörfern lebten und extreme Rituale der Unterschiedlichkeit praktizierten, etwa sich zu verstecken oder zu prostituieren, wenn ein Angehöriger einer höheren Statusgruppe (jeder andere) vorbei ging (Neary 1997; Pharr 1990: 76-77). Der offizielle Status dieser Menschen wurde 1871 endgültig abgeschafft; das führte zum Aufruhr und zu Ausschreitungen gegen sie überall in Japan, in einem Fall, in der südwestlichen Stadt Fukuoka, mit der Folge, dass 2200 ihrer Häuser niedergebrannt und viele von ihnen getötet wurden. Kurz nach dem „Befreiungs"-Gesetz von 1871 bezogen sich Regierungsdokumente auf die burakumin immer noch als ,die niedrigsten aller Menschen, fast wie Tiere" (siehe Pharr 1990: 77; Hane 1982: 146).

Heute gibt es immer noch zwischen zwei und drei Millionen burakumin, und sie stehen der Diskriminierung auf vielerlei Arten gegenüber. Zum Beispiel ist der Status der burakumin weiterhin so niedrig, dass eine Heirat mit einem Nicht-burakumin unwahrscheinlich ist. Vor einer Heirat wird heute in Japan gewöhnlich der Hintergrund der potentiellen Partner überprüft, ob sich eine Spur von burakumin-Abstammung findet; oft werden dafür Detekteien engagiert, die sich auf diese Arbeit spezialisiert haben. Ein Ergebnis davon ist laut einer Studie von 1967, dass bei den burakumin die Rate der Heiraten, die ausschließlich untereinander stattfinden, um die 90 Prozent beträgt (Hane 1982: 149). Die gleiche Praxis findet sich in der Anstellung neuer Arbeitnehmer durch große und kleine Unternehmen - auch hier wird der persönliche Hintergrund überprüft und werden potentielle Arbeitnehmer abgewiesen, wenn eine burakim-Abstammung aufgespürt wird. Während es neuerdings einen gewissen Anstieg bei den Verheiratungsraten mit Nicht-burakumin gibt und der Bildungsund Einkommenserwerb sich verbessert hat, besteht ein beträchtlicher Anteil der Diskriminierung dieser Menschen fort.

Die japanische Sprache. Das Japanische erlaubt dem Sprecher, verschiedene Ebenen von Rang, Formalität/Informalität und Respekt in einer komplexeren Art und Weise zu zeigen als irgendeine der westlichen Sprachen oder auch viele asiatische Sprachen. Ein Teil der japanischen Sprache, Sonkeigo genannt, wird benutzt, um Ehrung oder Formalität im Gespräch mit Höhergestellten auszu- 
drücken, während ein anderer Teil der Sprache, kenjogo, benutzt wird, um sich vor Höhergestellten demütig zu erniedrigen. Aber es gibt nicht nur verschiedene Worte, um Rang oder Formalität/Informalität anzuzeigen, es gibt auch verschiedene Partikeln und Verbformen, um eine von vielen Statusebenen auszudrücken. Goldstein und Tamura (1975) behaupten, dass Japanisch mehr als jede andere Sprache auf nicht-reziproken Verben, Substantiven, Adjektiven, Adverbien und Verbformen aufgebaut ist. Das bedeutet, dass die japanische Sprache dazu benutzt werden kann, Statusunterschiede und Status-Ehrerbietung in einer sehr komplexen Art und Weise herauszustellen ${ }^{3}$, und sie dazu auch benutzt wird.

Eine bedeutende Frage betrifft die Faktoren, die den relativen Status und die Formalität oder Informalität bei der verbalen Interaktion in Japan bestimmen. Studien legen nahe, dass Alter, Geschlecht, Beruf und die Zuordnung des Gegenübers zu Insidern oder Outsidern eine Rolle spielen (Goldstein/Tamura 1975). Collins (1975: 195) argumentiert, dass in den modernen industrialisierten Gesellschaften die Konversationsrituale informeller geworden seien. Der Gebrauch der japanischen Sprache zur Anzeige von Statusunterschieden und Formalität ist seit den Vorkriegsjahren um einiges zurückgegangen. Niemand hat jedoch behauptet, dass die Interaktionsrituale in Japan sich auch nur in die Nähe jener Informalität bewegt hätten, die sich in westlichen Industrienationen finden (siehe den Überblick über relevante Studien in Mouer/Sugimoto 1986: 351-352). Es kann daher mit Recht vertreten werden, dass die japanischen Konversationsrituale ein machtvolles Instrument dafür darstellen, die Statushierarchie in dieser Nation zu verstärken und zu perpetuieren.

Abschließende Bemerkungen zu Statusabstufungen in Japan. Die individualistische Voreingenommenheit der aktuellen Theorien sozialer Ungleichheit in postindustriellen Gesellschaften zeigt sich am deutlichsten in der allgemeinen Vernachlässigung der Status-Dimension wie auch bei Konzepten von Macht und Autorität. Diese sind beide Gemeingut der Gruppe. Sie werden von der Gruppe aus ihrem Kontext heraus definiert. Status-Ehre kann nur von den Gruppenmitgliedern in Abhängigkeit von den Werten der Gruppe erboten werden: Status

\footnotetext{
${ }^{3}$ Um das zu verdeutlichen, kömnen wir den Satz „Ich werde das für dich schneiden“ auf Japanisch benutzen. Die folgenden Zeilen drücken alle genau das aus, aber mit Veränderungen in den Worten, Verb-Endungen und Indikatoren für Status-Ehrerbietung, die den relativen Status des Sprechers zum Angesprochenen anzeigen - von der sehr formalen Ansprache eines vom Status her sehr hoch Gestellten in den oberen Sätzen bis zum sehr informellen Sprechen in den letzten Sätzen (Goldstein/Tamura 1975: 113): Watakushi ga o-kiri shite sashiagemasho; Watakushi ga o-kiri itashimasho; Watakushi ga o-kiri shimasho; Watashi ga o-kiri shimasho; Watashi ga kitte agemasho; Watashi ga kitte ageru way o (nur weibliche Sprecherinnen); Watashi ga kitte yaru wa yo (nur weibliche Sprecherinnen); Boku ga kitte ageru yo (nur männliche Sprecher); Boku ga kitte yaro (nur männliche Sprecher); Ore ga kitte yaru yo (nur männliche Sprecher); Ore ga kitte yaru sa zo (nur männliche Sprecher).
} 
kann man sich nicht einfach nehmen, wie Geld, Wohlstand und andere materielle Dimensionen von Stratifikation und Gütern. Studien haben gezeigt, dass selbst dann, wenn andere Arten von Ungleichheit in einer Gruppe mit weitgehendem Wertekonsens nicht vorliegen, Statusunterschiede wichtig werden (Della Fave/Hillery 1980). Das soll nicht heißen, dass die Vorteile einer Statusposition nicht durch Manipulation, Betrug oder Macht erreicht werden könnten: Man kann sie sich sicherlich in solcher Weise aneignen, und das wird oft der Fall sein.

Aber der entscheidende Punkt liegt darin, dass man zwar den Menschen ihr Geld nehmen kann, obwohl sie sich vollkommen klar darüber sind, dass es ihnen zu Unrecht genommen wird; um ihren Respekt oder die Vorteile einer Statusposition zu erringen, darf jedoch derjenige, der sie sich aneignet, die Menschen nicht wissen lassen, dass sie bei dem Vorgang manipuliert werden.

Macht, wie sie bei Weber unter utilitaristischen Annahmen definiert wird, findet sich reichlich in Asien genau wie im Westen, und sie wird hier wie dort auch reichlich genutzt. Aber folgt man Politikwissenschaftlern wie Pye (1985), scheint es, dass Weber die Art von Macht, die traditionell bei Herrschern in Asien festgestellt wird, nicht vollkommen bewusst war. Diese Art von Macht ist kombiniert mit Status und nur dann legitim, wenn der Machthaber sich an Regeln der Wohltätigkeit hält. Regeln der Wohltätigkeit können sich ändern. Das geschieht auch und verursacht Auseinandersetzung und Revolte. Solange jedoch diese Regeln der Gefolgschaft von ihrem Anführer aufrechterhalten werden, oder wenigstens aufrechterhalten zu werden scheinen, beugt sich nach der Tradition der meisten asiatischen Nationen die Gefolgschaft freiwillig und ohne negative Konnotation der Autorität (Pye 1985: X). In einer ähnlichen Kritik der individualistischen Vorannahmen hinter dem Konzept von Macht in westlichen Theorien untersuchte Hwang (1987) Kleingruppenexperimente in China und stellte fest, dass die Ergebnisse in "Macht-Spielen“ sich im Vergleich oft von den Ergebnissen solcher Experimente mit westlichen Menschen unterscheiden. Das führte zu der Folgerung, dass ,westliche Forschung zu zwischenmenschlichen Verhaltensmustern und Regeln des Austauschs die Vorannahme überwinden muss, Menschen seien isolierte Individuen, die dazu sozialisiert worden seien, rationale Entscheidungen auf der Basis von Eigeninteresse zu treffen" (nach Hwang 1987: 944).

Im Hinblick auf das spezifische Thema der sozialen Mobilität in Japan können wir deshalb in Frage stellen, ob die Standardmaße für soziale Mobilität, die über alle Industrieländer und sogar weniger entwickelte Nationen hinweg benutzt werden, hier genau passen. Welche Bewegung eines Sohnes von der beruflichen Position des Vaters zu einer anderen Position wird z. B. höher bewertet? Wäre es für den Sohn eines Vaters, der sich in der Position des Büro- 
Managers auf der mittleren Ebene eines kleinen Unternehmens befindet, die Bewegung zu einer Position als Partner in einer privaten Rechtsanwaltskanzlei, oder die Bewegung zu einer Position auf der mittleren Verwaltungsebene im Finanzministerium?

Während die Standardmaße für die berufliche Rangfolge, die im Westen entwickelt wurden und in vergleichenden Studien sozialer Mobilität verwendet werden, nahe legen würden, dass es ein größerer Schritt wäre, Anwalt zu werden, würden die meisten Japaner dem widersprechen mit dem Argument, dass dieser Sohn in beachtlich höherem Maße an Status und Achtbarkeit gewinne, wenn er eine Karriere im Finanzministerium machen könne. Oder ein anderes Beispiel: Würde die Position eines Sohnes als Rechtsanwalt in einer Abteilung von Sony im Vergleich zur Position seines Vaters als Rechtsanwalt in einer kleineren Firma als keine Intergenerationenmobilität gewertet werden? Das Überwiegen des Status des Unternehmens, in dem jemand beschäftigt ist, über die bloße berufliche Tätigkeit an sich und selbst über das Einkommen an sich würde viele Japaner zu dem Urteil führen, dass es für diesen Sohn eine beachtliche Intergenerationenmobilität gegeben habe.

\section{Schluss}

Ich möchte klarstellen, dass ich nicht gegen die in zunehmendem Maße standardisierte quantitative vergleichend-historische Forschung argumentiert habe, die heute die führenden soziologischen Fachzeitschriften durchzieht. Wir haben in den letzten Jahren viel erfahren über die Wahrscheinlichkeit oder Unwahrscheinlichkeit von ökonomischer Entwicklung und Verringerung der Armut in den weniger entwickelten Ländern - in ihrer Anbindung an die Weltwirtschaft. Gleichermaßen haben wir einiges darüber erfahren, welche Bedingungen die soziale Mobilität in den entwickelten Ländern auf der ganzen Welt fördern oder hemmen. Das sind nur zwei Beispiele für Forschungsfelder, die von den neuen Techniken der Statistik profitiert haben, die die Möglichkeiten für uns erweiterten, quantitative vergleichende und historische Forschungsmethoden anzuwenden. Was ich aber darlegen wollte, ist, dass in dem stürmischen Drang, diese neuen statistischen Werkzeuge zu benutzen und ,wissenschaftlicher" zu scheinen, der Wert der mehr Weberianischen qualitativen Annäherung an die vergleichende und historische Analyse in den letzten Jahren vernachlässigt wurde. Wir müssen uns eingestehen, dass Weber uns zu seiner Zeit mit Hilfe seines Ansatzes mehr beigebracht hat, als die aktuelle quantitative Forschung es getan hat. Wie oben schon bemerkt, lag Weber sicherlich im Hinblick auf vieles in Japan falsch. Aber hätte er in der Ära des „Jumbos“ gelebt, der Länder und Wissenschaftler immer näher zusammen brachte, wäre sein Einfluss auf unser Verständnis der Welt sogar noch größer gewesen. 
Wir könnten es so ausdrücken, dass der Unterschied zwischen den Methodologien der Unterschied zwischen dem Beschreiben eines Landes und dem Testen einer Theorie ist. In den multivariaten Modellen, die mit quantitativen und historischen Methoden entwickelt und getestet werden, verlieren wir die einzelnen Länder aus dem Blick. Wir erfahren lediglich, dass gewisse Variablen oder Kombinationen von Variablen wahrscheinlich diesen oder jenen Einfluss haben. Aber wir müssen einen Schritt zurücktreten und sozusagen einen oder zwei Bäume in dem Wald untersuchen. Manchmal können wir mehr über unsere Theorien erfahren, indem wir analysieren, warum ein bestimmtes Land oder eine bestimmte Gruppe von Ländern nicht zu unseren Theorien passt, wenn es so aussieht, als handele es sich dabei um Ausreißer. Indem wir das tun, können wir Effekte und Variablen entdecken, an die wir vorher vielleicht nicht gedacht haben. Wir können dann Maße und Indikatoren für diese vorher unerkannten Variablen entwickeln, sie in unsere Theorien einfügen und dann mit den quantitativen Forschungsmethoden fortfahren, um tragfähigere Modelle zu entwickeln für so etwas wie ökonomische Entwicklung, soziale Mobilität, Geschlechterungleichheit usw. Gute Beispiele hierfür sind die Fallstudien zur ökonomischen Entwicklung oder $\mathrm{zu}$ deren Fehlen in südostasiatischen Ländern und im Falle von Japan wichtige Dimensionen sozialer Ungleichheit in postindustriellen Gesellschaften. Soweit Max Weber jedoch Recht hatte, können qualitative vergleichende und historische Methodologien vielleicht zeigen, dass es einzigartige Kombinationen historischer Kräfte und aktueller Bedingungen gibt, welche es fast unmöglich machen, einige Länder in Vergleiche einzubeziehen, wenn es um so etwas wie das Wesen ihres Schichtungssystems oder die Gründe ihrer ökonomischen Entwicklung oder deren Fehlens geht. Aber in den meisten Fällen bedeutet das nicht, dass unsere multivariaten Modelle, die durch den abwechselnden Gebrauch von qualitativen und quantitativen Methoden entwickelt wurden, uns nicht einer hundertprozentigen Erklärung der Varianz immer näher bringen würden.

\section{Literatur}

Alderson, A. S., F. Nielsen, 2002: Globalization and the Great U-Turn: Income Inequality Trends in 16 OECD Countries, in: American Journal of Sociology 107, 12441299.

Alderson, A. S., F. Nielsen, 1999: Income Inequality, Development, and Dependence: A Reconsideration, in: American Sociological Review 64, 606-631.

Alletzhauser, A. J., 1990: The House of Nomura: The Inside Story of the Legendary Japanese Financial Dynasty, New York: Arcade.

Bellah, R., 1985: Tokugawa Religion: The Cultural Roots of Modern Japan, New York: Free Press. 
Bornschier, V., C. Chase-Dunn, 1985: Transnational Corporations and Underdevelopment, New York: Praeger.

Clark, R., 1971: Einstein: The Life and Times, New York: Times Mirror World Publishing.

Collins, R., 1997: Religious Economy and the Emergence of Capitalism in Japan, in: American Sociological Review 62, 843-865.

Collins, R., 1975: Conflict Sociology, New York: Academic Press.

Colignon, R. A., C. Usui, 2003: Amakudari: The Hidden Fabric of Japan's Economy, Ithaca, New York: Cornell University Press.

Della Fave, L. R., G. Hillery, 1980: Status Inequality in a Religious Community: The Case of a Trappist Monastery, in: Social Forces 59, 62-84.

De Vos, G., H. Wagatsuma, 1966: Japan's Invisible Race: Caste in Culture and Personality, Berkeley: University of California Press.

Dukas, H., B. Hoffman, 1979: Albert Einstein: The Human Side, New Glimpses from His Archives, Princeton, N.J.: Princeton University Press.

Durkheim, E., 1951: Suicide, New York: Free Press.

Eisenstadt, S. N., 1996: Japanese Civilization: A Comparative View, Chicago: University of Chicago Press.

Erikson, R., J. H. Goldthorpe, 1992: The Constant Flux: A Study of Class Mobility in Industrial Societies, Oxford: Clarendon.

Firebaugh, G., 1996: Does Foreign Capital Harm Poor Nations? New Estimates Based on Dixon and Boswell's Measures of Capital Penetration, in: American Journal of Sociology 102, 563-578.

Firebaugh, G., 1992: Growth Effects of Foreign and Domestic Investment, in: American Journal of Sociology 98, 105-130.

Frank, A. G., 1998: ReOrient: Global Economy in the Asian Age, Los Angeles: University of California Press.

Friedrichs, R., 1970: A Sociology of Sociology, New York: Free Press.

Girling, J. L. S., 1981: Thailand: Society and Politics, Ithaca, N.J.: Cornell University Press.

Gluck, C., 1985: Japan's Modern Myths: Ideology in the Late Meiji Period, Princeton, N. J.: Princeton University Press.

Goldstein, B., K. Tamura, 1975: Japan and America: A Comparative Study in Language and Culture, Tokyo: Tuttle.

Golzio, K. H., 1985: Max Weber on Japan: The Role of the Government and the Buddhist Sects, in: A. E. Buss (Hrsg.), Max Weber in Asian Studies, Leiden: E. J. Brill, S. 90-101.

Gouldner, A., 1970: The Coming Crisis in Western Sociology, New York: Basic Books.

Gouldner, A., 1973: For Sociology: Renewal and Critique in Sociology Today, New York: Basic Books.

Grusky, D., R. Hauser, 1984: Comparative Social Mobility Revisited: Models of Convergence and Divergence in 16 Countries, in: American Sociological Review 49, $19-38$. 
Halliday, J., 1975: A Political History of Japanese Capitalism, New York: Monthly Review Press.

Hane, M., 1982: Peasants, Rebels, and Outcasts: The Underside of Modern Japan, New York: Pantheon.

Hertz, T., 2004: Rags, Riches and Race: The Intergenerational Economic Mobility of Black and White Families in the United States, in: S. Bowles, H. Gintis, M. Osborne (Hrsg.), Unequal Chances: Family Background and Economic Success, New York: Russell Sage and Princeton University Press.

Hwang, K., 1987: Face and Favor: The Chinese Power Game, in: American Journal of Sociology 92, 944-974.

Jansen, M. B., 2000: The Making of Modern Japan, Cambridge Mass.: Belknap/Harvard University Press.

Johnson, C., 1982: MITI and the Japanese Miracle, Stanford, Calif.: Stanford University Press.

Kentor, J., T. Boswell, 2003: Foreign Capital Dependence and Development: A New Direction, in: American Sociological Review 68, 301-313.

Kerbo, H., 2006a: World Poverty: Global Inequalities and the Modern World System, New York: McGraw-Hill.

Kerbo, H., 2006b: Social Stratification and Inequality, New York: McGraw-Hill.

Kerbo, H., 1981: Characteristics of the Poor: A Continuing Focus in Social Research, in: Sociology and Social Research 65, 323-331.

Kerbo, H. R., J. A. McKinstry, 1995: Who Rules Japan?: The Inner-Circles of Economic and Political Power, Westport, Conn.: Praeger.

Keyes, C. F., 1989: Thailand: Buddhist Kingdom as Modern Nation-State, Boulder, Colo.: Westview Press.

Koh, B. C., 1989: Japan's Administrative Elite, Berkeley: University of California Press.

Kuhn, T., 1962: The Structure of Scientific Revolutions, Chicago: University of Chicago Press.

Kuhn, T., 1970: The Structure of Scientific Revolutions, 2. A., Chicago: University of Chicago Press.

Levathes, L., 1994: When China Ruled The Seas, New York: Oxford University Press.

Moller, S., D. Bradley, E. Huber, F. Nielsen, J. Stephens, 2003: Determinants of Relative Poverty in Advanced Capitalist Democracies, in: American Sociological Review $68,22-51$.

Mouer, R., Y. Sugimoto, 1986: Images of Japanese Society, London: Kegan Paul International.

Neary, I., 1997: Burakumin in Contemporary Japan, in: M. Weiner (Hrsg.), Japan's Minorities: The Illusion of Homogeneity, London: Routledge, S. 50-78.

Park, Y. H., 1986: Bureaucrats and Ministers in Contemporary Japanese Government, Berkeley: University of California, Institute of East Asian Studies.

Pharr, S. J., 1990: Losing Face: Status Politics in Japan, Berkeley: University of California Press.

Pye, L. W., 1985: Asian Power and Politics: The Cultural Dimensions of Authority, Cambridge, Mass.: Belknap Press/Harvard University Press. 
Ragin, C. C., 2000: Fuzzy-Set Social Science, Chicago: University of Chicago Press.

Ragin, C. C., D. Zaret, 1983: Theory and Method in Comparative Research: Two Strategies, in: Social Forces 61, 731-754.

Reischauer, E. O., 1987: The Japanese, Cambridge, Mass.: Harvard University Press.

Reischauer, E. O., A. M. Craig, 1978: Japan: Tradition and Transformation, New York: Houghton Mifflin.

Smelser, N. J., 1976: Comparative Methods in the Social Sciences, Englewood Cliffs, N.J.: Prentice Hall.

Strasser, H., 1976: The Normative Structure of Sociology: Conservative and Emancipatory Themes in Social Thought, London: Routledge \& Kegan Paul.

Weltbank, 2000: World Development Report 2000/2001, New York: Oxford University Press.

Wright, E. O., 1997: Class Counts: Comparative Studies in Class Analysis, Cambridge: Cambridge University Press.

Wyatt, D. K., 1984: Thailand: A Short History, New Haven: Yale University Press.

Yawata, Y., 1963: Religionssoziologische Untersuchungen zur Geschichte Japans, in: R. König, J. Winckelmann (Hrsg.), Max Weber zum Gedächtnis, Köln/Opladen: Westdeutscher Verlag (Kölner Zeitschrift für Soziologie and Sozialpsychologie, Sonderheft 7) 